\title{
Multimodal Investigation of Chiton Stylus Reveals New Biomineral
}

\author{
Linus Stegbauer ${ }^{1}$, Paul Smeets ${ }^{1}$, Esen Ercan Alp ${ }^{2}$ and Derk Joester ${ }^{1}$
}

${ }^{1}$ Northwestern University, Evanston, Illinois, United States, ${ }^{2}$ Advanced Photon Source, Sector 3, Argonne National Laboratory, Lemont, Illinois, United States

Biominerals are broadly used to reinforce structural materials in organisms, enabling for instance locomotion, feeding, and defense, but also finding application in manipulation of light and in sensing of acceleration and magnetic fields.[1] A defining principle of biomineralized tissues is their composite nature, reaping synergy from the combination of a soft macromolecular matrix and a hard, inorganic mineral phase. The capability of organisms to functionally grade such composites by precisely controlling the phase, size, shape, orientation, dispersion, and spatial distribution of mineral nanoparticles remains unmatched. Chitons, a class of marine mollusks belong to the most prolific mineralizers, They are probably best known for the extreme hardness, rich chemistry, and intricate phase assemblage of their radula teeth.[2-4]

The chiton radula is bilaterally symmetrical, slender ribbon with transverse rows teeth (Figure 1). Two outsize major lateral teeth make contact with the surfaces that chitons graze on. Major lateral teeth consist of a tooth head and a stylus that is anchored in the radula membrane. The function of the radula requires highly disparate material properties. The tooth head that scrapes over the hard substratum is comprised of a highly mineralized cusp with exceptional hardness, wear resistance, and self-sharpening properties. The cusp is supported by a softer core, and the entire head is mounted on the stylus, an L-shaped structure that is integral to the complex movement of the tooth head during the feeding stroke. As the stylus ultimately controls the orientation of the tooth with respect to the substratum, and therefore the efficiency of the scraping action, its stiffness is intermediate to the staff tooth head and the flexible radula.

The stylus is generally described as an unmineralized tissue, even though a considerable amount of biomineral precursors are known to be transported through the stylus during mineralization of the tooth head. It has been proposed that large-capacity iron binding proteins, in combination with nucleation and/or growth inhibitors may be present to prevent mineralization.[2] However, the chemical form in which iron appears in the the junction zone and stylus remains unclear. We therefore set out to map the redox state and chemical environment of iron in the tooth of Cryptochiton stelleri, with the long-term goal to trace these parameters over the development of the radula and thus gain insight into the mechanisms at play. Given the complex shape and small size of chiton teeth, and the extraordinarily rich chemistry of iron, determining the spatial distribution of multiple, often poorly crystalline phases requires techniques that combine high spatial resolution with sensitivity for subtle differences in oxidation state and coordination geometry of iron. Classical Mößbauer spectroscopy offers the latter, and has been used to study the overall phase assemblage, but is strictly a bulk technique.[5] Synchrotron Mößbauer spectroscopy (SMS), however, recently emerged as a powerful tool that combines high spatial resolution with the deep insights offered by classical MS.[6] Herein, we report on our discovery of a new biomineral in the mature upper stylus, using SMS and correlative imaging and spectroscopy technicques (Figure 2).[7] 

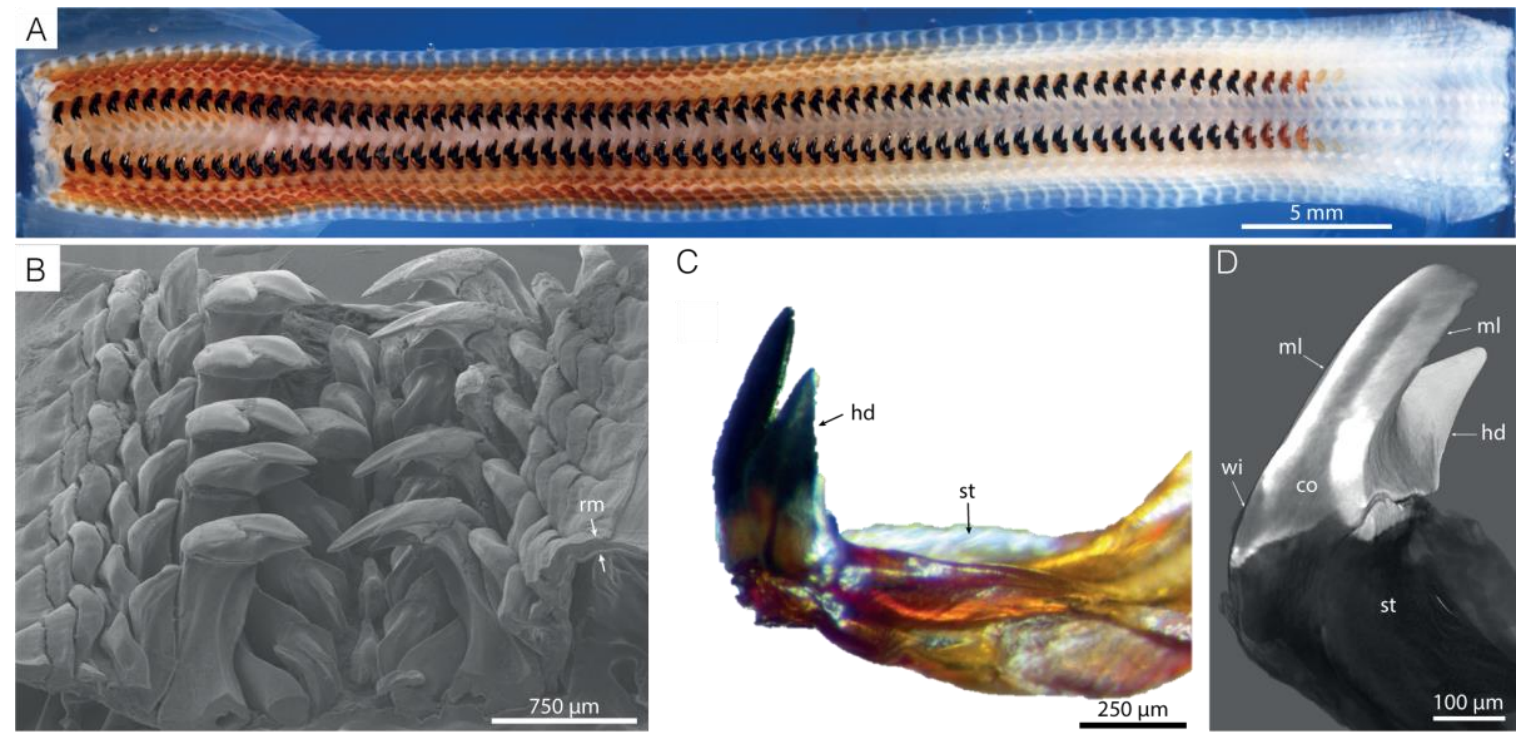

Figure 1. Figure 1. Anatomy of the radula of C. stelleri. A. Whole radula with mature teeth at the anterior end (left) and newly formed teeth prior to mineralization at the posterior end (right). B. SEM image of the anterior end of the radula. Major lateral teeth consist of the tricuspidate head (hd) and the stylus (sty). The stylus anchors teeth on the thin $(<100 \mu \mathrm{m})$ and flexible radula membrane (rm). The stylus canal (stc) runs along the length of the stylus but terminates below the head. C. Extracted tooth with head and upper stylus. The black color is due to the presence of magnetite (Fe3O4) D. Rendering of a longitudinal slice of a reconstruction of the normalized linear attenuation (LAC) coefficient of a tooth head (hd) and upper stylus (st). Note the LAC is highest for the outer magnetite layer of the head (ml), intermediate for AFP-based composite of the core (co), and rather low in the stylus. Typical for Cryptochiton type teeth, there is a window (wi) in the magnetite layer on the trailing (anterior) side of the head.
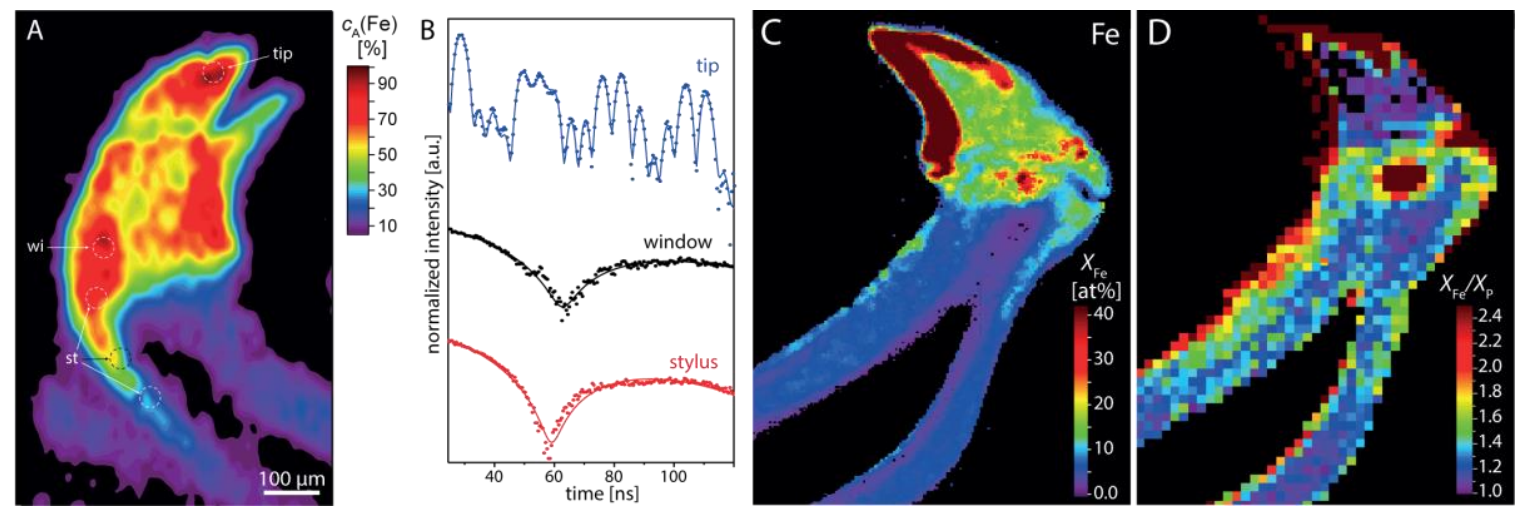

Figure 2. Figure 2. Characterization of the upper stylus. A. Map of the normalized area concentration of iron, based on SMS resonance intensity, across a longitudinal section of a mature tooth head and upper stylus. The section was oriented similar to the reconstruction in (Figure 1D). B. SMS point spectra taken at the locations in the tip (blue circles), the window (black circles), and the stylus (red circles) indicated in (B), and models fit to the data (solid lines of corresponding color). C-D.Longitudinal section of the tooth and upper stylus. SEM-EDS elemental map reporting the mole fraction of $\mathrm{Fe}(\mathrm{C})$ and the ratio of iron and phosphorous (D). Maps were downsampled to ensure that the uncertainty of the Fe/P ratio is less that $10 \%$, based on counting statistics. 


\section{References}

[1] Lowenstam, H. A.; Weiner, S. On biomineralization; Oxford University Press: New York, 1989.

[2] Joester, D.; Brooker, L. R. in Iron Oxides: From nature to applications; Faivre, D., Ed.; Wiley-VCH Verlag GmbH \& Co. KGaA: Weinheim, Germany, 2016; pp 177-206.

[3] Gordon, L. M.; Joester, D. Nature 2011, 469, 194-197.

[4] Weaver, J. C.; Wang, Q.; Miserez, A.; Tantuccio, A.; Stromberg, R.; Bozhilov, K. N.; Maxwell, P.; Nay, R.; Heier, S. T.; DiMasi, E. et al. Analysis of an ultra hard magnetic biomineral in chiton radular teeth. Materials Today 2010, 13, 42-52.

[5] St. Pierre, T. G.; Evans, L. A.; Webb, J, Hyperfine Interactions 1992, 71, 1275-1278.

[6] Yan, L.; Zhao, J.; Toellner, T. S.; Divan, R.; Xu, S.; Cai, Z.; Boesenberg, J. S.; Friedrich, J. M.; Cramer, S. P.; Alp, E. E, J Synchrotron Rad 2012, 19, 814-820.

[7] This work was in part supported by NSF (DMR-1508399, DMR-1905982). LS was supported by a research fellowship of the Deutsche Forschungsgemeinschaft (STE2689/1-1). 